"(C) 2015 IEEE. Personal use of this material is permitted. Permission from IEEE must be obtained for all other uses, in any current or future media, including reprinting/republishing this material for advertising or promotional purposes, creating new collective works, for resale or redistribution to servers or lists, or reuse of any copyrighted component of this work in other works." 


\title{
Analytic Performance Model for State-Based MAC Layer Cooperative Retransmission Protocols
}

\author{
Brett Hagelstein, Member, IEEE, Mehran Abolhasan, Senior Member, IEEE, \\ Daniel Franklin, Member, IEEE, Farzad Safaei, Senior Member, IEEE, and Wei Ni, Member, IEEE
}

\begin{abstract}
Cooperative retransmission can significantly improve link reliability over lossy and time-varying wireless links. However, comparing retransmission protocols is challenging, and generally requires simplistic assumptions specific to each protocol. In this paper, we develop a general model to evaluate cooperative retransmission protocols with distributed, slot-based contention algorithms. Specifically, we propose to calculate the relay time-out probabilities at a MAC time-slot scale, formulate retransmission outcomes as functions of the time-out probabilities, and derive the probability of a retransmission process for every data frame. We also propose a Markov extension of our model to characterise the dependency between retransmissions of multiple frames. This enables our model to analyse continuous retransmissions of successive frames. Validated by QualNet simulations, our model can analytically predict the probabilities of cooperative retransmissions with an accuracy of $\pm 1 \%$. As a result, direct comparisons between cooperative retransmission protocols become tangible, without implementing the full protocol in a state-based simulator.
\end{abstract}

Index Terms-cooperative retransmission, opportunistic retransmission, IEEE 802.11, MAC, ARQ, CMAC, DAFMAC, $\triangle$-MAC, PRO

\section{INTRODUCTION}

Cooperative retransmission is a well-established technique for improving wireless network capacity [1]. Its effectiveness is strongly dependent on the choice of relay selection algorithm, as this determines both the probability of retransmission success and the probability of collisions between simultaneous retransmission attempts. Consequently, relay selection algorithm design remains an open area of research.

There are two fundamentally different approaches to cooperative retransmission. One approach is for either the source [2], [3] or destination [4] to nominate a specific node to act as a relay; alternatively, a contending set of relays can use a distributed algorithm to autonomously attempt to select a single relay [5]-[8]. Each approach has its own merits, however, as yet there is no general analytic method which can be used to comprehensively compare the performance of

B. Hagelstein, M. Abolhasan and D. Franklin are with the Centre for Real-time Information Networks, University of Technology, Sydney, NSW 2007, Australia. e-mail: \{brett.hagelstein, mehran.abolhasan, daniel.franklin\}@uts.edu.au

F. Safaei is with the Information and Communication Technology Research Institute, University of Wollongong, Wollongong, NSW 2522, Australia. email: farzad_safaei@uow.edu.au

W. Ni is with the Digital Productivity and Service (DP\&S) Flagship, CSIRO, Sydney, NSW 2122, Australia. email: wei.ni@csiro.au

This manuscript was submitted on September 1, 2014. This research was partly supported by Australian Research Council grant DP0879507 "Novel Architecture for Next Generation Wireless Relay Networks" different techniques. Such a technique would be extremely valuable in determining the appropriateness of different protocols for a particular scenario, identifying potential performance limitations and quickly evaluating new ideas for cooperative retransmission strategies.

A direct comparison of the cooperative algorithms is challenging, due to the limitations and narrow focus of specific protocols, as well as restrictive assumptions made in the protocols. Following are a number of examples of the assumptions made in the literature.

- The relay is assumed to always receive the source frame and it successfully forwards the frame without error [9];

- A relay is assumed to always be at the optimal location midway between the source and destination nodes [10];

- It is assumed there are no retransmission collisions [11];

- Provided that retransmissions do not collide, it is assumed that they always succeed [12];

- Relays are assumed to never collide, but instead are sufficiently synchronised that any retransmissions during the same slot will combine to improve the frame SNR at the destination [13];

- There is a transitional data frame decoding probability, but all RTS, CTS and ACK control frames are assumed to be correctly decoded [3]; and

- All links are assumed to have an identical probability of transmission success [6], [8], [14], [15].

Even where these assumptions are valid, as each example uses a different set of assumptions, a meaningful comparison between protocols becomes intractable. Development of a more general model which removes some or all of these assumptions is therefore important.

In this paper, we propose a general model to analytically evaluate cooperative retransmission schemes, where a detailed failure mode analysis is established and behaviours of distributed slot-based contentions are captured. Retransmission performances can be characterised by the probability of each possible outcome from a retransmission attempt. The outcomes include retransmission success, as well as failure caused by data frame corruption, ACK corruption, retransmission collision or no relay availability. We calculate the relay time-out probabilities at a MAC time-slot scale, and formulate each of the outcomes as a function of the relay time-out probabilities. We explicitly derive the probability of the outcome, which is able to analytically characterise the cooperative retransmission process of every data frame.

Our proposed model can further be extended to practical environments, where collisions of cooperative retransmissions 
can be significantly reduced by exploiting the time coherence of wireless channels. Specifically, the previous successful relay can be elected as a preferred relay to retransmit a new data frame in a dedicated contention-free time slot. The proposed model can establish a Markov process for the election of preferred relays, and identify the outcome probabilities by solving the preferred relay Markov model. As a result, continuous retransmissions of successive data frames can be analysed.

Confirmed by QualNet simulations, the proposed analytic model is able to accurately evaluate a range of retransmission protocols, including the (non-cooperative) plain 802.11 ARQ [16], CMAC [9], DAFMAC [17], $\triangle$-MAC [3] and PRO [6] retransmission protocols. Potential relay node $i$ may select a specific delay $t_{i}$ [6], [17], or it may randomly decide whether or not to contend for a given slot at the beginning of that slot [11]. The proposed model accommodates both strategies. In this sense, our proposed model is suitable for both contentionbased networks, such as IEEE 802.11 WiFi and IEEE 802.15.4 Zigbee, and contention-free multi-hop networks.

The proposed model is novel because of the depth of analysis offered; to date, no other models have been proposed which include a complete failure mode analysis with independent link parameters. The proposed model is a significant advance of the state of the art. It provides a quantitative measure of the efficacy of any given retransmission scheme. The model also provides a practical means to accurately compare diverse cooperative retransmission protocols in an arbitrary scenario. It can be used as a prediction tool, and operate in real-time to choose the adequate protocol adapting to changing network conditions.

The remainder of this paper is structured as follows. Cooperative retransmission timing probabilities for the five popular protocols, i.e., 802.11 ARQ, CMAC, DAFMAC, $\triangle$-MAC, and PRO, are derived in Section II. The general modelling of retransmission outcomes is derived in Section III. The Markov extension of the proposed model is developed in Section IV. The analytic model is validated against a detailed protocol simulation in Section V. The performance of individual cooperative protocols is compared in Section VI, followed by discussions on practical applications in Section VII. Finally, conclusions are provided in Section VIII.

List of notations used in this paper:

$\mathcal{N}_{n} \quad$ set of all neighbouring nodes

$\mathcal{N}_{p} \quad$ set of nodes participating in cooperation

$\mathcal{N}_{c} \quad$ set of nodes contending for retransmission

$i \quad$ a relay node $i \in \mathcal{N}_{c}$

$t_{i} \quad$ cooperative back-off time for $i$

$T_{\max } \quad$ number of slots in back-off window

$X_{i} \quad$ uniform random value, $X_{i} \in[0,1)$

$p_{i t}(t) \quad$ probability that $i$ 's timer expires in slot $t$

$p_{i b}(t) \quad$ probability that $i$ 's timer expired before slot $t$

$p_{i a}(t) \quad$ probability that $i$ 's timer expires after slot $t$

$P_{D}(s, d)$ probability of successful data transmission

$P_{A}(d, s)$ probability of successful ACK transmission

$F_{i} \quad$ scoring function for $i$ in DAFMAC
$F_{\max } \quad$ maximum score for active relays in DAFMAC

$F_{\min }$ minimum score for active relays in DAFMAC

$L_{i} \quad$ link-dependent delay for $i$ in DAFMAC

$a_{X} \quad$ random delay weight for DAFMAC, $a_{X} \in[0,1]$

$W_{i}\left(\mathcal{N}_{c}, t\right)$ probability $i$ wins retransmission contention in slot $t$ given the contending set $\mathcal{N}_{c}$

$p_{\mathcal{N}_{c}} \quad$ probability that only set $\mathcal{N}_{c}$ receives transmission

$\mathcal{S}_{c} \quad$ set of all possible contending sets $\mathcal{N}_{c} \subseteq \mathcal{S}_{c}$

$P(a, b) \quad$ probability of preferred state changing from $a$ to $b$

Q Markov process's infinitesimal generator matrix

$\pi \quad$ stationary distribution of Markov process

$\pi_{i} \quad$ probability that $i$ is the preferred relay

\section{Cooperative Retransmission Protocols And TIME-OUT PROBABILITIES}

Cooperative retransmission protocols employ the distributed timer method (originally proposed in [1]) to activate relaying in a decentralised manner. Each cooperative node that successfully decoded a frame from the source set up a random backoff timer. The node starts to count down, if it does not receive an ACK from the intended destination. The node retransmits the frame if the timer becomes zero. If the retransmission is successful, the destination returns an ACK and all the cooperative relays flush the frame from their buffers.

Failed cooperative transmissions are handled differently by each protocol; some protocols continue cooperative retransmissions until successful, while others use a singleretransmission limit. For consistency in this analysis, we calculate the probability of a successful cooperative retransmission after a single attempt.

The nodes may use channel state information available at the MAC layer, such as received signal strength (RSS) and packet delivery ratio (PDR), to estimate the probability of successfully relaying the frame to the destination. Those with a high likelihood of success can set small back-off timers, and subsequently retransmit with a short delay. Assume that the RSS or historical PDR values are known to the node, and remain valid throughout the observation period. This assumption is reasonable and practical, because each node can measure its own RSS. Each node can also calculate its own PDR by accumulating the number of frames it has delivered (through monitoring the acknowledgements of the destination). As a result, our proposed model that will be presented in Sections III and IV is suitable to different types of networks.

Let $\mathcal{N}_{n}$ denote the set of neighbour nodes with non-zero PDR to both source and destination. The participating set $\mathcal{N}_{p}$ defines the neighbouring nodes that have elected to act as relay candidates. The contending set $\mathcal{N}_{c}$ denotes the set of participating relays which receive the source frame. Note that hidden nodes can affect the protocols where multiple relays can contend for a retransmission opportunity, such as CMAC, DAFMAC and PRO. To eliminate the issue, it is specified that $\mathcal{N}_{p}$ consists of neighbouring relays that can hear each other. This can be achieved by running routing/flooding protocols before cooperative retransmissions. It is also specified that each cooperative relay retransmits at most once for a frame, in case the relay is a hidden node to the destination. 

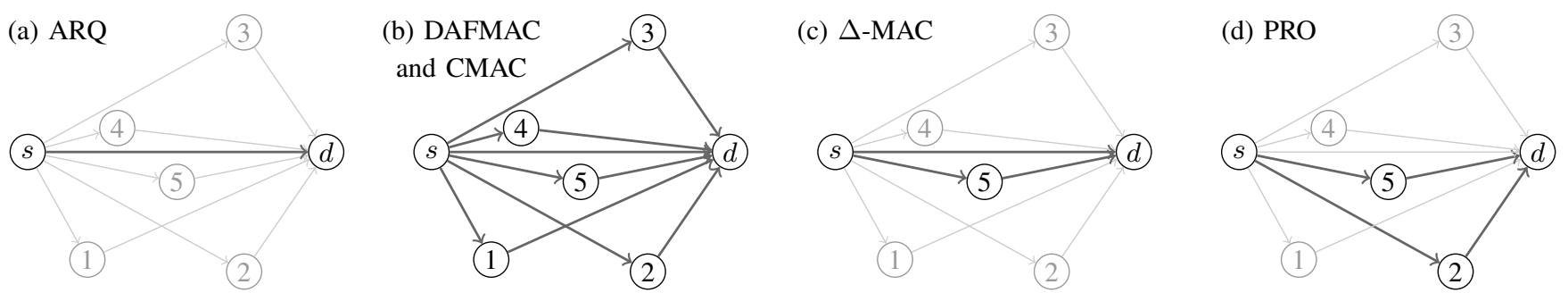

Fig. 1: Examples of the participating relay set for (a) plain IEEE 802.11 ARQ, (b) DAFMAC and CMAC, (c) $\triangle$-MAC, and (d) PRO.

A relay $i \in \mathcal{N}_{c}$ with a pre-selected back-off timer $t_{i}$ has three possible states; namely, the timer may expire before $\left(t_{i}<\right.$ $t$ ), during $\left(t_{i}=t\right)$, or after $\left(t_{i}>t\right)$ a given time-slot $t$. We define the probabilities of being in each state as $p_{i b}(t), p_{i t}(t)$, and $p_{i a}(t)$, respectively. Clearly, we have

$$
p_{i b}(t)+p_{i t}(t)+p_{i a}(t)=1 .
$$

Let $P_{D}(a, b)$ denote the probability of a successful transmission from node $a$ to $b$. Also let $P_{A}(b, a)$ denote the ACK transmission success probability from $b$ to $a$.

We consider four popular cooperative retransmission protocols; namely, CMAC, DAFMAC, $\triangle$-MAC and PRO, indicated by the superscripts ${ }^{C}, D, \Delta$ and ${ }^{P}$, respectively. We also consider the non-cooperative $802.11 \mathrm{ARQ}$, indicated by the superscript ${ }^{A}$, for comparison purpose. The time-out probabilities of the protocols are derived, which are important to analyse the protocols, as will be discussed in Section III.

\section{A. 802.11 Automatic Repeat Request (ARQ)}

We start with the original non-cooperative 802.11 ARQ protocol, where only the source node retransmits [16], as shown in Figure 1; hence $\mathcal{N}_{p}^{A}=\{s\}$. This is represented in the model by letting $P_{D}(s, i)=1$ (i.e. a perfect channel) while $P_{D}(i, d)$ remains scenario-specific. $T_{\max }^{A}$ is the upper bound of the contention window size from which $t_{i}^{A}$ is randomly selected, as given by

$$
t_{i}^{A}=\left\lfloor X_{i} T_{\max }^{A}\right\rfloor
$$

where $X_{i} \sim \operatorname{Un}[0,1)$. Using (2), the probability of the ARQ timer $t_{i}^{A}$ expiring before time slot $t$ is:

$$
\begin{aligned}
p_{i b}^{A}(t) & =\operatorname{Pr}\left\{\left\lfloor T_{\max }^{A} X_{i}\right\rfloor<t\right\} \\
& =\operatorname{Pr}\left\{X_{i}<\frac{t}{T_{\max }^{A}}\right\}=\frac{t}{T_{\max }^{A}} .
\end{aligned}
$$

The probability that $t_{i}^{A}$ expires after slot $t$ is:

$$
\begin{aligned}
p_{i a}^{A}(t) & =\operatorname{Pr}\left\{\left\lfloor T_{\max }^{A} X_{i}\right\rfloor>t\right\} \\
& =\operatorname{Pr}\left\{X_{i} \geq \frac{t+1}{T_{\max }^{A}}\right\}=1-\frac{t+1}{T_{\max }^{A}} .
\end{aligned}
$$

From (1), (3) and (4), the probability $t_{i}^{A}$ expires in slot $t$ is:

$$
p_{i t}^{A}(t)=1-p_{i b}^{A}(t)-p_{i a}^{A}(t)=\frac{1}{T_{\max }^{A}} .
$$

These functions are intuitive and are plotted in Figure 2 for visual reference. The $p_{i t}^{A}(t)$ value is constant over $t$ because the

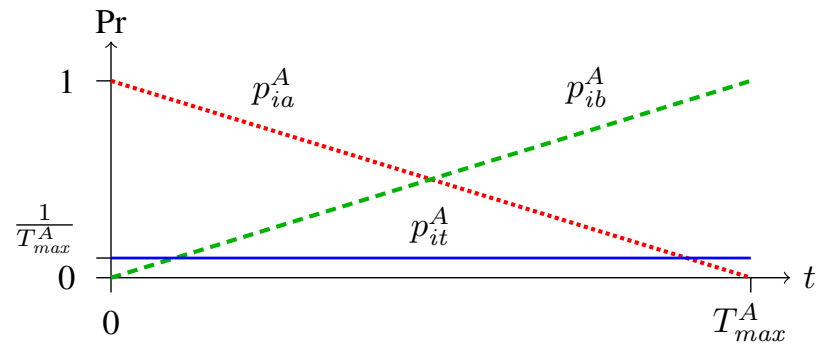

Fig. 2: Visualisation of the slot probabilities of ARQ derived in (3), (4) and (5), CMAC and $\Delta$-MAC have similar plots

timeout probability is equal for all time-slots. The probability of the timer expiring before this time-slot $p_{i b}^{A}(t)$ monotonically increases with $t$ and the probability of expiring after this time slot $p_{i a}^{A}(t)$ monotonically decreases with $t$.

\section{B. CMAC}

The CMAC protocol is one of the earliest IEEE 802.11based cooperative retransmission protocols. All the relays which overhear and successfully decode the data frame enter a CSMA/CA back-off period to contend for retransmission. The participating set of cooperative relays also includes the source node. $\mathcal{N}_{p}^{C}=\left\{s, \mathcal{N}_{n}\right\}$. Each contending relay $i \in \mathcal{N}_{p}^{C}$ generates a random back-off time, as given by

$$
t_{i}^{C}=\left\lfloor X_{i} T_{\max }^{C}\right\rfloor .
$$

We can obtain the probabilities of the CMAC back-off timer expiring before, during, and after time slot $t$, as given by (3), (4), and (5), due to the analogy of (6) to (2).

\section{DAFMAC}

In the DAFMAC relay selection algorithm, all neighbour nodes participate in cooperative retransmissions, as shown in Figure 1. The relay nodes generate their contention delay based on their instantaneous RSS [5]. The nodes with higher RSS will have shorter delays than other nodes. A random delay component is added to all delay calculations to avoid collisions from the nodes with similar link quality.

The general form of the DAFMAC contention delay is:

$$
t_{i}^{D}=\left\lfloor\left(\left(1-a_{X}\right)\left(\frac{F_{i}-F_{\max }}{F_{\min }-F_{\max }}\right)+a_{X} X_{i}\right) T_{\max }^{D}\right\rfloor
$$

where $a_{X}$ is the random delay weighting, $F_{i}$ is the scoring function for node $i$, and $F_{\min }$ and $F_{\max }$ are the outputs of 
the scoring function with the lowest and highest expected RSS values, respectively. For analytical tractability, we consider $a_{X} T_{\max }^{D}>1$.

The probability that DAFMAC timer $t_{i}^{D}$ expires before slot $t$ can be given by

$$
\begin{aligned}
p_{i b}^{D}(t) & =\operatorname{Pr}\left\{\left\lfloor L_{i}^{D}+a_{X} X_{i} T_{\max }^{D}\right\rfloor<t\right\} \\
& =\operatorname{Pr}\left\{X_{i}<\frac{t-L_{i}^{D}}{a_{X} T_{\max }^{D}}\right\} \\
& = \begin{cases}0, & t<L_{i}^{D} \\
\frac{t-L_{i}^{D}}{a_{X} T_{\max }^{D}}, & t \in\left[L_{i}^{D}, L_{i}^{D}+a_{X} T_{\max }^{D}\right) \\
1, & t \geq L_{i}^{D}+a_{X} T_{\max }^{D} .\end{cases}
\end{aligned}
$$

where $L_{i}^{D}=\left(1-a_{X}\right)\left(\frac{F_{i}-F_{\max }}{F_{\min }-F_{\max }}\right) T_{\max }^{D}$ represents the 'link quality' component which remains static during the retransmission process.

Likewise, the probability that DAFMAC timer $t_{i}^{D}$ expires after slot $t$ can be given by

$$
\begin{aligned}
p_{i a}^{D}(t) & =\operatorname{Pr}\left\{\left\lfloor L_{i}^{D}+a_{X} X_{i} T_{\max }^{D}\right\rfloor>t\right\} \\
& =\operatorname{Pr}\left\{X_{i} \geq \frac{t+1-L_{i}^{D}}{a_{X} T_{\max }^{D}}\right\} \\
& = \begin{cases}1, & t<L_{i}^{D}-1 \\
1-\frac{t+1-L_{i}^{D}}{a_{X} T_{\max }^{D}}, & t \in\left[L_{i}^{D}-1, L_{i}^{D}+a_{X} T_{\max }^{D}-1\right) \\
0, & t \geq L_{i}^{D}+a_{X} T_{\max }^{D}-1 .\end{cases}
\end{aligned}
$$

The probability that a DAFMAC retransmission delay times out in slot $t$ can therefore be given by

$$
\begin{aligned}
p_{i t}^{D}(t) & =1-p_{i b}^{D}(t)-p_{i a}^{D}(t) \\
& = \begin{cases}0, & t<L_{i}^{D}-1 \\
\frac{t+1-L_{i}^{D}}{a_{X} T_{\max }^{D}}, & t \in\left[L_{i}^{D}-1, L_{i}^{D}\right) \\
\frac{1}{a_{X} T_{\max }^{D}}, & t \in\left[L_{i}^{D}, L_{i}^{D}+a_{X} T_{\max }^{D}-1\right) \\
1-\frac{t-L_{i}^{D}}{a_{X} T_{\max }^{D}}, & \\
t \in\left[L_{i}^{D}+a_{X} T_{\max }^{D}-1, L_{i}^{D}+a_{X} T_{\max }^{D}\right) \\
0, & t \geq L_{i}^{D}+a_{X} T_{\max }^{D} .\end{cases}
\end{aligned}
$$

These probabilities are piecewise linear functions and are plotted in Figure 3. There is a finite region where the timeout probability is non-zero which may be unique to each contending relay. This region determined by the relative value of the link quality component of the delay and the weighting of the random delay component. This region also forms the transition bounds of the $p_{i b}^{A}(t)$ and $p_{i a}^{A}(t)$ probabilities.

\section{D. $\triangle-M A C$}

In the $\triangle$-MAC retransmission algorithm, one relay is nominated by the source node prior to transmission [3]. The relay has the highest joint PDR to the source and the destination.

The relay monitors the destination. For every frame the source sends, the relay conducts decoding. In the case where

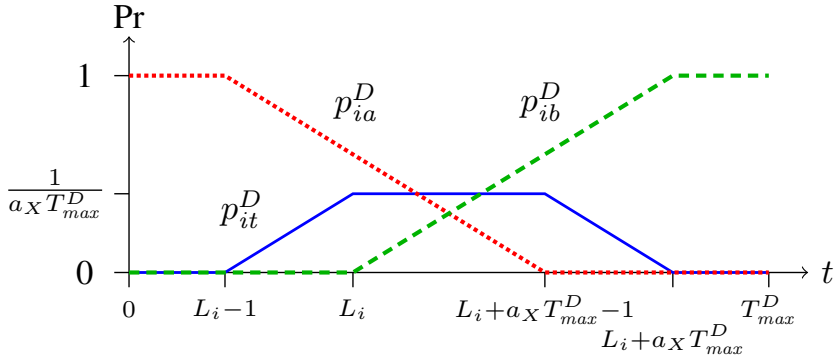

Fig. 3: Visualisation of the slot probabilities of DAFMAC derived in (7), (8) and (9), where $a_{X} T_{\max }^{D}>1$

the relay has successfully decoded the frame while the destination has not returned an ACK, the relay sends an ACK to the source. Upon receiving this ACK, the source stops ARQ retransmissions and waits for the retransmissions by the relay.

The $\Delta$-MAC participating set consists of the source and the nominated relay. $\mathcal{N}_{p}^{\Delta}=\{s, i\}$. Their time-out probabilities can be presented by (3), (4), and (5). This is because only one of the source and the nominated relay is activated to transmit in $\triangle$-MAC, and the activated node generates the back-off timer in the same way as the ARQ. The time-out probabilities can be plotted, as shown in Figure 2.

\section{E. $P R O$}

In the PRO algorithm, neighbour nodes are ranked using $R S S_{i, d}$, with $R S S_{s, i}$ used to resolve a tie [6]. Relays are added to the participating set until the cumulative joint retransmission probability reaches a threshold $T h_{r}$, as shown in Figure 1. $\left(T h_{r}=0.95[6]\right)$. The participating set, $\mathcal{N}_{p}^{P} \subseteq \mathcal{N}_{n}$, is known to all neighbours from control signalling. PRO produces contention windows as such that more highly ranked relays have a smaller contention window $T_{\max }^{P}(i)$, where $T_{\max }^{P}(i)$ is the contention window size for node $i$.

$$
T_{\max }^{P}(i)=2^{\min \left(\left\lfloor\frac{i+9}{2}\right\rfloor, 10\right)}
$$

where $i$ is the ordered participating node index (from 1 to $\left.\left|\mathcal{N}_{p}^{P}\right|\right)$. The contention delay for node $i$ is given by

$$
t_{i}^{P}=\left\lfloor T_{\max }^{P}(i) X_{i}\right\rfloor .
$$

Unlike other algorithms, individual PRO relays uses different $T_{\max }^{P}$ values. The total range of potential delays is given by

$$
t_{i}^{P} \in\left[0,2^{\min \left(\left\lfloor\frac{\left|\mathcal{N}_{p}^{P}\right|+9}{2}\right\rfloor, 10\right)}-1\right] .
$$

The probability of the PRO timer $t_{i}^{P}$ expiring before time slot $t$ is bounded by the $T_{\max }^{P}(i)$ contention range:

$$
p_{i b}^{P}(t)= \begin{cases}\frac{t}{T_{\max }^{P}(i)}, & t \in\left[0, T_{\max }^{P}(i)-1\right] \\ 1, & t \in\left(T_{\max }^{P}(i)-1, T_{\max }^{P}\left(\left|\mathcal{N}_{p}^{P}\right|\right)-1\right] .\end{cases}
$$




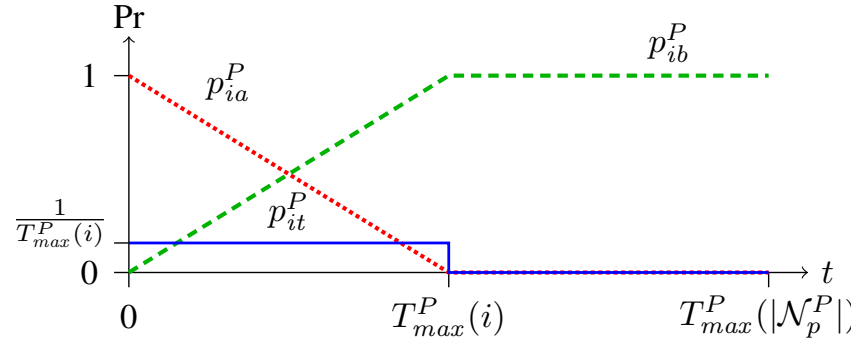

Fig. 4: Visualisation of the slot probabilities of PRO derived in (10), (11) and (12), illustrated where $i<\left|N_{p}^{P}\right|$

Similarly to (4), the probability that $t_{i}^{P}$ expires after $t$ is:

$p_{i a}^{P}(t)= \begin{cases}1-\frac{t+1}{T_{\max }^{P}(i)}, & t \in\left[0, T_{\max }^{P}(i)-1\right] \\ 0, & t \in\left(T_{\max }^{P}(i)-1, T_{\max }^{P}\left(\left|\mathcal{N}_{p}^{P}\right|\right)-1\right] .\end{cases}$

From (1), (10), (11), the probability $t_{i}^{P}$ expires in slot $t$ is:

$$
\begin{aligned}
p_{i t}^{P}(t) & =1-p_{i b}^{P}(t)-p_{i a}^{P}(t) \\
& = \begin{cases}\frac{1}{T_{\max }^{P}(i)}, & t \in\left[0, T_{\max }^{P}(i)-1\right] \\
0, & t \in\left(T_{\max }^{P}(i)-1, T_{\max }^{P}\left(\left|\mathcal{N}_{p}^{P}\right|\right)-1\right] .\end{cases}
\end{aligned}
$$

Figure 4 plots the PRO time-out probability functions. The timeout probability $p_{i t}^{P}(t)$ is uniformly spread over the range $\left[0, T_{\max }^{P}(i)-1\right]$. If a third or subsequent relay is participating in the retransmission, it contends using a larger contention window. The cooperative contention of higher-ranked relays will be resolved at the conclusion of the shorter window, as shown in the figure.

\section{ANALYSES ON COOPERATIVE RETRANSMISSIONS OF \\ A Single DATA FRAME}

In this section, we evaluate the performance of the protocols, given $p_{i a}(t), p_{i t}(t)$, and $p_{i b}(t)$. The key metrics that we evaluate include the end-to-end success probability of a retransmission, the probability of retransmission collisions, and the probabilities of retransmission failures due to unsuccessful retransmission and unsuccessful acknowledgement. They are the probabilities of possible outcomes of a retransmission attempt. For analytical tractability, we assume that each contending set retransmits the frame at most once, after which all relays discard the frame.

First, the probability that any node from any contending relay set successfully retransmits the frame to the destination and the ACK is successfully received can be given by

$$
\begin{aligned}
& \operatorname{Pr}\{\text { success }\}= \\
& \sum_{\mathcal{N}_{c} \subseteq \mathcal{S}_{c}} \sum_{i \in \mathcal{N}_{c}} \sum_{t=0}^{T_{\max }-1} p_{\mathcal{N}_{c}} W_{i}\left(\mathcal{N}_{c}, t\right) P_{D}(i, d) P_{A}(d, s)
\end{aligned}
$$

where "success" indicates the event of successful delivery of a data frame in a retransmission attempt, $p_{\mathcal{N}_{c}}=$ $\prod_{i \in \mathcal{N}_{c}} P_{D}(s, i) \prod_{i \in \mathcal{N}_{p} \backslash \mathcal{N}_{c}}\left(1-P_{D}(s, i)\right)$ is the probability that of the participating set, the devices in set $\mathcal{N}_{c}$ received and decoded the frame from the source while others did not; $W_{i}\left(\mathcal{N}_{c}, t\right)=p_{i t}(t) \prod_{j \in \mathcal{N}_{c} \backslash\{i\}} p_{j a}(t)$ is the probability that relay $i$ wins the cooperative retransmission contention because all other relays time out after it.

The sum operation of $\sum_{t=0}^{T_{\max }-1}$ accounts for the successful delivery over the entire contention time slots; the sum operation of $\sum_{i \in \mathcal{N}_{c}}$ accounts for the successful delivery by any relay in the contending set; and the sum operation $\sum_{\mathcal{N}_{c} \subseteq \mathcal{S}_{c}}$ accounts for all possible contending sets.

Detailed derivation of (13) is provided in Appendix I.

If $\operatorname{Pr}\{$ success $\}>P_{D}(s, d)$, the corresponding cooperative retransmission scheme will result in a higher probability of frame delivery compared to a single (source) node ARQ retransmission.

Similarly, we can derive the probability that the destination receives the data frame via retransmission, but the source fails to receive the ACK, as given by

$\operatorname{Pr}\{$ Afail $\}=$

$\sum_{\mathcal{N}_{c} \subseteq \mathcal{S}_{c}} \sum_{i \in \mathcal{N}_{c}} \sum_{t=0}^{T_{\max }-1} p_{\mathcal{N}_{c}} W_{i}\left(\mathcal{N}_{c}, t\right) P_{D}(i, d)\left(1-P_{A}(d, s)\right)$

where "A fail" indicates the event that the source fails to decode the ACK from the destination following a retransmission attempt of the relays.

We can also derive the probability of any node winning contention but failing to deliver the data frame to the destination, as given by

$$
\begin{aligned}
& \operatorname{Pr}\{\text { D fail }\}= \\
& \sum_{\mathcal{N}_{c} \subseteq \mathcal{S}_{c}} \sum_{i \in \mathcal{N}_{c}} \sum_{t=0}^{T_{\max }-1} p_{\mathcal{N}_{c}} W_{i}\left(\mathcal{N}_{c}, t\right)\left(1-P_{D}(i, d)\right)
\end{aligned}
$$

where " $D$ fail" indicates the event that the destination fails to decode the data frame of a retransmission attempt of the relay.

We can also calculate the probability of an empty contending set (i.e. $\left|\mathcal{N}_{c}\right|=0$ ), as given by

$$
\operatorname{Pr}\{\text { no relays }\}=\prod_{i \in \mathcal{N}_{p}}\left(1-P_{D}(s, i)\right)
$$

which is because no participating relay receives the source frame. Here, "no relays" indicates the event that no contending relay is active at a retransmission time slot.

We proceed to derive the collision probability of cooperative retransmissions. There are three cases where time slot $t$ is free from collision. The first case is that a node timer has expired before this time-slot. The second case is that all node timers will expire after this slot. The last case is that only a single node timer expires in this slot. As a result, we can derive the probability of collision, as given by

$$
\begin{aligned}
\operatorname{Pr}\{\text { collision }\}= & 1-\sum_{\mathcal{N}_{c} \subseteq \mathcal{S}_{c}} \sum_{t=0}^{T_{\max }-1}\left(\left(1-\prod_{i \in \mathcal{N}_{c}}\left(1-p_{i b}(t)\right)\right)\right. \\
& \left.+\prod_{i \in \mathcal{N}_{c}} p_{i a}(t)+\sum_{i \in \mathcal{N}_{c}} W_{i}\left(\mathcal{N}_{c}, t\right)\right) p_{\mathcal{N}_{c}}(17)
\end{aligned}
$$

where "collision" indicates the event that a collision occurs 
due to retransmission attempts of multiple relays within a time slot. The three terms in the brackets correspond to the three collision-free cases with regards to time slot $t$, respectively. $W_{i}\left(\mathcal{N}_{c}, t\right)=p_{i t}(t) \prod_{j \in \mathcal{N}_{c} \backslash\{i\}} p_{j a}(t)$ is the probability that relay $i$ wins contention in time-slot $t$ without collision, as described earlier.

The computational complexity of our proposed model is low. Calculations of (13) to (17) just involve operations of real scalar values. Our model can readily support more devices. In a case of 20 relays (i.e., $\left|\mathcal{S}_{c}\right|=20$ ) and $T_{\max }=16$, a total of $16 \times \sum_{i=1}^{20}\left(\begin{array}{c}20 \\ i\end{array}\right) i=167772160$ terms are added up in (13), and each term is a product of four real scalar values. The complexity is about $6.7 \times 10^{8}$ float point operations (FLOPs). Such complexity can be implemented instantly with many commercially available computers. (For example, a single-core $2.5 \mathrm{GHz}$ processor has a theoretical performance of $10 \times 10^{9}$ FLOPs/sec.)

\section{Proposed Markov Model For CoOperative RETRANSMISSIONS OF CONTINUOUS FRAMES}

In many practical environments, a currently successful relay is likely to be selected again for the retransmission of the next data frame, due to the time coherence of wireless channels. Retransmission collisions can be significantly reduced by allowing the relay to transmit in a contention-free period [17]. We refer to the relay as a preferred relay, and enable it to immediately retransmit a failed frame in time-slot 0 . The remaining relays contend in the interval $t_{i} \in\left[1, T_{\max }-1\right]$.

Figure 5 shows the flowchart of the election of a preferred relay, where the DAFMAC protocol is considered for illustration purpose. It is shown that the election can be accomplished in a distributed manner. Specifically, a relay can set a preferred status flag after it successfully retransmitted a frame. Other nodes maintain their default (non-preferred) status. The relay immediately loses the preferred status, if it fails to decode a frame from the source or to deliver the frame to the destination (by monitoring acknowledgements). From the network perspective, the outcomes of the distributed election process include that the current preferred relay remains preferred, or there is a new preferred relay, or there is no preferred relay for the retransmission of the next frame. The outcomes are respectively highlighted in green, pink, and blue in the figure.

The election process of preferred relays cannot be captured by the analysis focused on a single retransmission process, as described in Section III. This is because the retransmissions of successive frames become dependent if preferred relays are elected based on previous retransmissions, whereas analysis in Section III is based on the assumption of independent retransmissions of different frames.

We propose to characterise the preferred relay process as a Markov chain model with a total of $\left(\left|\mathcal{N}_{p}\right|+1\right)$ states, where $\left|\mathcal{N}_{p}\right|$ states correspond to the cases with one preferred relay and the other state corresponds to the case with no preferred relay. An example state machine of the proposed Markov model is shown in Figure 6 for a system with three participating nodes. There are five unique state transitions: stay with preferred relay $i$, switch from no preferred relay to some

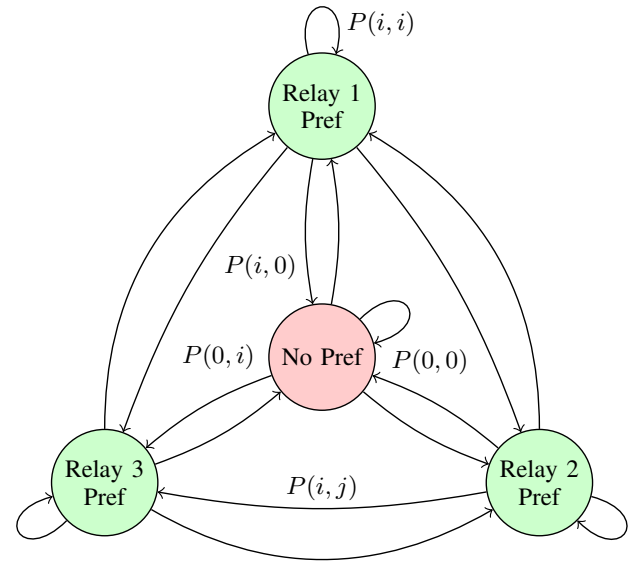

Fig. 6: State diagram and transition probability notation with three nodes

preferred relay $i$, change preferred relay from $i$ to $j$, switch from preferred relay $i$ to no preferred relay, or stay with no preferred relay. The probabilities of each transition are denoted as $P(i, i), P(0, i), P(i, j), P(i, 0)$, and $P(0,0)$ respectively.

\section{A. Derivation of Markov Transition Probabilities}

The probability of the preferred relay changing from $a$ to $b$ is denoted as $P^{c}(a, b)$ during a retransmission attempt, and as $P^{n}(a, b)$ during a non-cooperative frame (i.e., a successful transmission of the source). The total state transition probability is therefore the weighted sum of the probabilities, as given by

$$
P(a, b)=\left(1-P_{D}(s, d)\right) P^{c}(a, b)+P_{D}(s, d) P^{n}(a, b) .
$$

1) Transition Probability $P(i, i)(i \neq 0)$ : The preferred node $i$ must receive the data frame and successfully retransmit it to the destination to remain preferred. The probability of this is:

$$
P^{c}(i, i)=P_{D}(s, i) P_{D}(i, d)
$$

Node $i$ must receive the frame to maintain preferred status, even when cooperation is not required:

$$
P^{n}(i, i)=P_{D}(s, i)
$$

Hence, the total probability of $i$ keeping preferred status is:

$$
\begin{gathered}
P(i, i)=\left(1-P_{D}(s, d)\right) P_{D}(s, i) P_{D}(i, d) \\
+P_{D}(s, d) P_{D}(s, i)
\end{gathered}
$$

2) Transition Probability $P(0, i)(i \neq 0)$ : When there is no initially preferred relay, any participating relay $i$ can potentially retransmit and become preferred. By definition, $i$ must receive the source frame to become preferred, as illustrated in Figure 5. Node $i$ must then win contention ahead of any other node and successfully retransmit the data frame to the destination.

Let $\mathcal{N}_{c \bar{i}}$ be the set of contending relay combinations excluding $i$, and $\mathcal{S}_{\bar{c} \bar{i}}$ be the set of all possible contending relays 


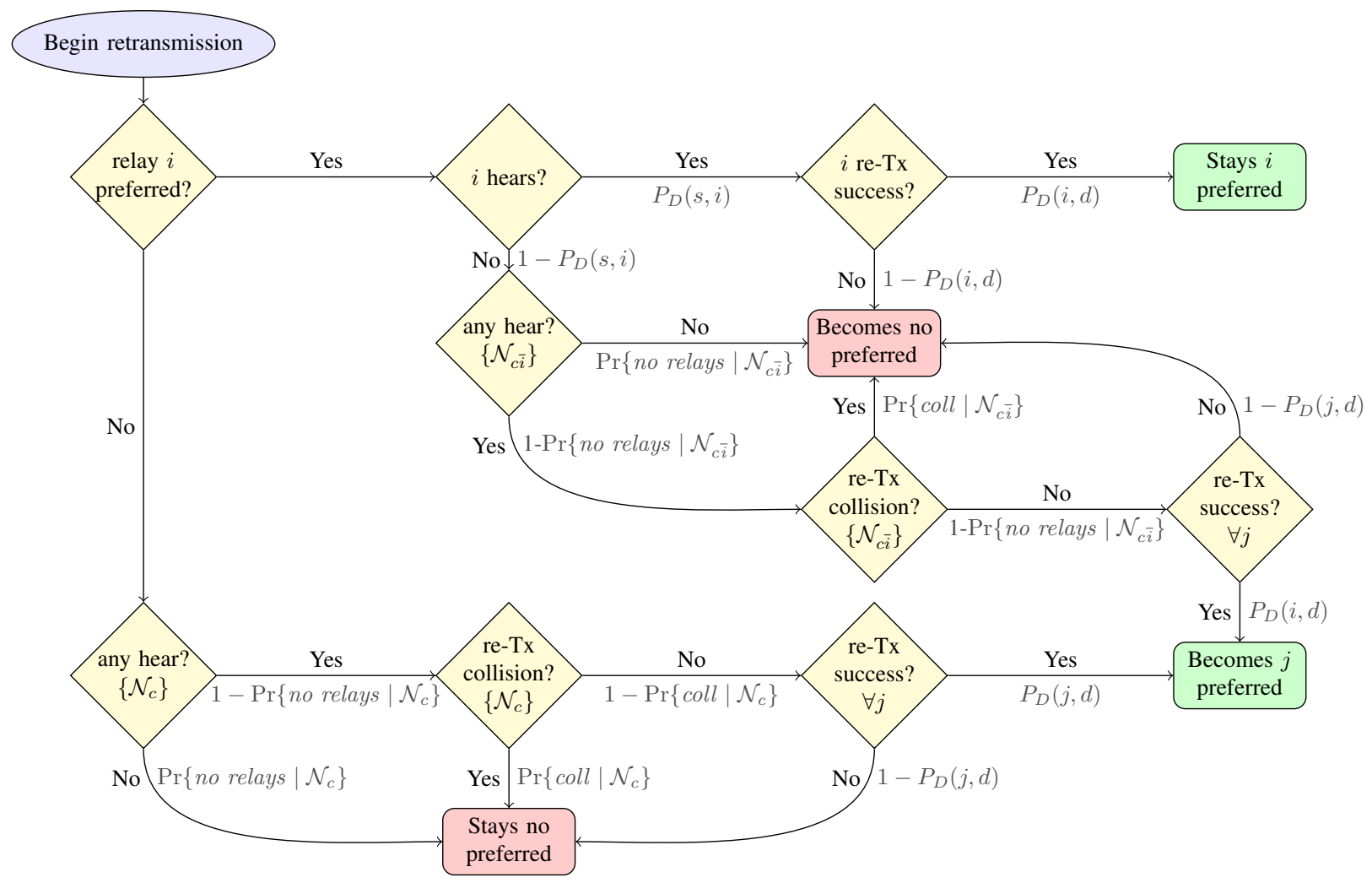

Fig. 5: Retransmission process with a potential preferred relay

excluding $i$. Therefore:

$$
P^{c}(0, i)=P_{D}(s, i) P_{D}(i, d) \sum_{\mathcal{N}_{c \bar{i}} \in \mathcal{S}_{c \bar{i}}} \sum_{t=0}^{T_{\max }-1} W_{i}\left(\mathcal{N}_{c}, t\right) p_{\mathcal{N}_{c \bar{\imath}}}
$$

where $W_{i}\left(\mathcal{N}_{c}, t\right)$ can be calculated by using the time-out probabilities updated for the preferred relay case. In the presence of the preferred relay $i$, the contention delay function with the preferred relays can be given by

$t_{i}^{D}=\left\{\begin{array}{rr}0, & i \text { is preferred } \\ \left\lfloor 1+\left(\left(1-a_{X}\right)\left(\frac{F_{i}-F_{\max }}{F_{\min }-F_{\max }}\right)+a_{X} X_{i}\right)\right. & \left.\left(T_{\max }^{D}-1\right)\right\rfloor, \\ \text { otherwise. }\end{array}\right.$

Clearly, the preferred relay does not require the time-out probabilities, as it retransmission takes place in the contentionfree time-slot 0 . For the remaining relays, we can redefine

$$
L_{i}^{D}=1+\left(1-a_{X}\right)\left(\frac{F_{i}-F_{\max }}{F_{\min }-F_{\max }}\right)\left(T_{\max }^{D}-1\right) .
$$

and their time-out probabilities follow (7), (8), and (9).

On the other hand, a node cannot gain the preferred status without successfully retransmitting the frame, therefore:

$$
P^{n}(0, i)=0
$$

Hence the total probability is given by:

$$
\begin{aligned}
P(0, i)=(1 & \left.-P_{D}(s, d)\right) P_{D}(s, i) P_{D}(i, d) \\
& \times \sum_{\mathcal{N}_{c \bar{i}} \in \mathcal{S}_{c \bar{i}}} \sum_{t=0}^{T_{\max }-1} W_{i}\left(\mathcal{N}_{c}, t\right) p_{\mathcal{N}_{c \bar{i}}}
\end{aligned}
$$

3) Transition Probability $P(i, j)(i \neq j$ and $i, j \neq 0)$ : In the case of transfer of the preference from one relay to another, the current preferred relay must fail to decode a frame while another node succeeds in doing so. That node then successfully retransmits to the destination.

Assume $i$ is the initial preferred relay and $j$ becomes the new preferred relay. Let $\mathcal{N}_{c \overline{i j}}$ denote the set of contending nodes excluding $i$ and $j$, and let set $\mathcal{S}_{c \overline{i j}}$ denote all possible combinations of contending nodes other than $i$ and $j$. Relays $i$ and $j$ are excluded from the iterative set calculations because their status is known. Following the flowchart provided in Figure 5, the probability of transferring preference from $i$ to $j$ can be given by

$$
\begin{aligned}
P^{c}(i, j)= & P_{D}(s, j)\left(1-P_{D}(s, i)\right) P_{D}(j, d) \\
& \times \sum_{\mathcal{N}_{c \bar{i} \in} \in \mathcal{S}_{c \bar{i}}} \sum_{t=0}^{T_{\max }-1} W_{j}\left(\mathcal{N}_{c \bar{i}}, t\right) p_{\mathcal{N}_{c \overline{i j}}}
\end{aligned}
$$

Preferred status cannot transfer from one node to another without a retransmission attempt, therefore: $P^{n}(i, j)=0$. 
The total probability is then given by:

$$
\begin{aligned}
P(i, j)=(1 & \left.-P_{D}(s, d)\right) P_{D}(s, j)\left(1-P_{D}(s, i)\right) P_{D}(j, d) \\
& \times \sum_{\mathcal{N}_{c \overline{i j}} \in \mathcal{S}_{c \overline{i j}}} \sum_{t=0}^{T_{\max }-1} W_{j}\left(\mathcal{N}_{c \bar{i}}, t\right) p_{\mathcal{N}_{c \overline{i j}}}
\end{aligned}
$$

4) Transition Probability $P(i, 0)(i \neq 0): P^{c}(i, 0)$ is solved by noting that the total probability flux out of any state is 1 , therefore:

$$
P^{c}(i, 0)=1-P^{c}(i, i)-\sum_{j \in \mathcal{N}_{c \bar{i}}} P^{c}(i, j)
$$

There is a finite probability that a node will lose preferred status after a direct transmission if the preferred node does not receive the frame:

$$
P^{n}(i, 0)=1-P_{D}(s, i)
$$

The total transition probability is therefore:

$$
\begin{aligned}
P(i, 0)=( & \left.-P_{D}(s, d)\right)\left(1-P^{c}(i, i)-\sum_{j \in \mathcal{N}_{c \bar{i}}} P^{c}(i, j)\right) \\
& +P_{D}(s, d)\left(1-P_{D}(s, i)\right)
\end{aligned}
$$

5) Transition Probability $P(0,0)$ : The system will remain with no preferred relay; if no relays received the source frame, if there is a collision, or if the destination fails to decode the frame. This value is not needed to solve the state probabilities for the Markov process.

\section{B. Markov Modelling and State Probabilities}

The state transition probabilities are used to create the infinitesimal generator matrix, $\mathbf{Q}$, to solve the Markov process [18]. For the example network with three potential relays:

$$
\mathbf{Q}=\left(\begin{array}{cccc}
-\sum_{i=0, i \neq 0}^{3} P(0, i) & P(0,1) & P(0,2) & P(0,3) \\
P(1,0) & -\sum_{i=0, i \neq 1}^{3} P(1, i) & P(1,2) & P(1,3) \\
P(2,0) & P(2,1) & -\sum_{i=0, i \neq 2}^{3} P(2, i) & P(2,3) \\
& & P(3,2) & -\sum_{i=0, i \neq 2}^{3} P(3, i)
\end{array}\right)
$$

There is insufficient information to determine a unique solution. However, we know that the sum of all probabilities must be 1 . Therefore, this can be placed in the the lower row of the transposed matrix. Now:

$$
\mathbf{Q}_{n}^{T}=\left(\begin{array}{cccc}
-\sum_{i=0, i \neq 0}^{3} P(0, i) & P(1,0) & P(2,0) & P(3,0) \\
P(0,1) & -\sum_{i=0, i \neq 1}^{3} P(1, i) & P(2,1) & P(3,1) \\
P(0,2) & P(1,2) & -\sum_{i=0, i \neq 2}^{3} P(2, i) & P(3,2) \\
1 & 1 & 1 & 1
\end{array}\right)
$$

which has the unique solution vector:

$$
\mathbf{e}_{n}=\left(\begin{array}{llll}
0 & 0 & 0 & 1
\end{array}\right)^{T},
$$

where the superscript ${ }^{T}$ denotes transpose.

Therefore, solving the linear equation:

$$
\mathbf{Q}_{n}^{T} \boldsymbol{\pi}=\mathbf{e}_{n}
$$

for the $\pi$ vector computes the proportion of the time spent in each state, that is the probability that relay $i$ is preferred, where the first index calculates the probability of no preferred relay.

The computational complexity of inverting $\mathbf{Q}_{n}^{T}$ to solve $\boldsymbol{\pi}$ is tractable. The dimension of $\mathbf{Q}_{n}$ is $\left(\left|\mathcal{N}_{c}\right|+1\right) \times\left(\left|\mathcal{N}_{c}\right|+1\right)$. Take a popular Gaussian elimination method to invert $\mathbf{Q}_{n}^{T}$. The complexity is about $\frac{2}{3}\left(\left|\mathcal{N}_{c}\right|+1\right)^{3}$ FLOPs. In the case of 20 active relays $\left|\mathcal{N}_{c}\right|=20$, a total of only 6714 FLOPs are required.

\section{Probabilities of Retransmission Outcomes}

There is a probability $\pi_{i} P_{D}(s, i)$ that relay $i$ is preferred, receives the source frame and retransmits without contention. There is also a probability $\pi_{i}\left(1-P_{D}(s, i)\right)$ that $i$ was preferred, but fails to receive the data frame and the remaining nodes contend for retransmission. This contending set is defined as:

$$
\mathcal{N}_{c \bar{i}} \triangleq\left\{\mathcal{N}_{p} \cap\{i\}\right\}^{\prime} .
$$

The probability of retransmission success is the summation:

$$
\begin{aligned}
& \operatorname{Pr}\{\text { pref success }\} \triangleq \pi_{0} \operatorname{Pr}\{\text { success }\} \\
& \quad+\sum_{i \in \mathcal{N}_{p}} \pi_{i} P_{D}(s, i) P_{D}(i, d) P_{A}(d, s) \\
& \quad+\sum_{i \in \mathcal{N}_{p}} \pi_{i}\left(1-P_{D}(s, i)\right) \operatorname{Pr}\left\{\text { success } \mid \mathcal{N}_{\bar{c} \bar{i}}\right\}
\end{aligned}
$$

The probability of data frame failures with preferred relays is:

$$
\begin{aligned}
& \operatorname{Pr}\{\text { pref } D \text { fail }\} \triangleq \pi_{0} \operatorname{Pr}\{D \text { fail }\} \\
& \quad+\sum_{i \in \mathcal{N}_{p}} \pi_{i} P_{D}(s, i)\left(1-P_{D}(i, d)\right) \\
& \quad+\sum_{i \in \mathcal{N}_{p}} \pi_{i}\left(1-P_{D}(s, i)\right) \operatorname{Pr}\left\{D \text { fail } \mid \mathcal{N}_{c \bar{i}}\right\}
\end{aligned}
$$

The probability of ACK frame failure with preferred relays is:

$$
\begin{aligned}
& \operatorname{Pr}\{\text { pref } A \text { fail }\} \triangleq \pi_{0} \operatorname{Pr}\{\text { A fail }\} \\
& \quad+\sum_{i \in \mathcal{N}_{p}} \pi_{i} P_{D}(s, i) P_{D}(i, d)\left(1-P_{A}(d, s)\right) \\
& \quad+\sum_{i \in \mathcal{N}_{p}} \pi_{i}\left(1-P_{D}(s, i)\right) \operatorname{Pr}\left\{A \text { fail } \mid \mathcal{N}_{c \bar{i}}\right\}
\end{aligned}
$$

The probability for a contention collision is only non-zero for non-preferred retransmissions:

$$
\begin{aligned}
\operatorname{Pr}\{\text { pref coll }\} & \triangleq \pi_{0} \operatorname{Pr}\{\text { coll }\} \\
& +\sum_{i \in \mathcal{N}_{p}} \pi_{i}\left(1-P_{D}(s, i)\right) \operatorname{Pr}\left\{\text { coll } \mid \mathcal{N}_{c \bar{i}}\right\} .
\end{aligned}
$$




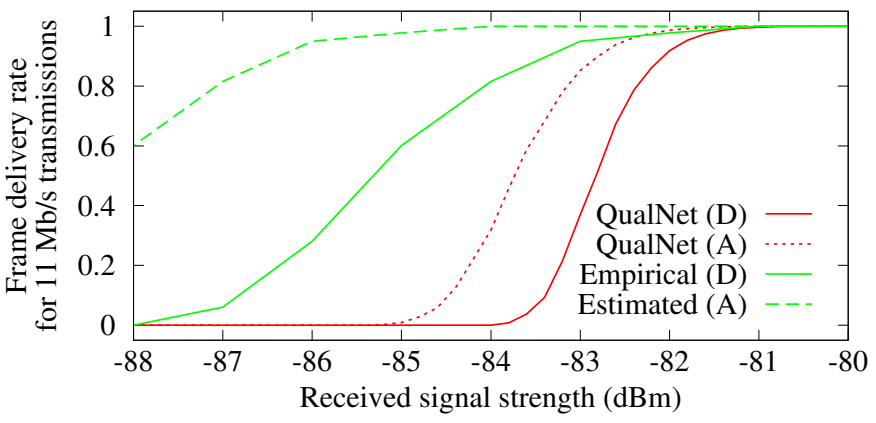

Fig. 7: The RSS-PDR relationship obtained from QualNet for IEEE $802.11 b$.

\section{Model Validation}

This section validates the analytic cooperative retransmission model by comparing its predictions with QualNet simulations. An example scenario is described in detail, simulated using an integrated PHY/MAC layer implementation of each of the evaluated protocols in QualNet [19], and evaluated analytically using the proposed model. The results predicted by the analytic model precisely match the simulation results.

\section{A. Link Quality Relationship}

The analytic retransmission model is specifically designed to be independent of the propagation or physical layer model. For model verification, the required RSS-PDR relationship is obtained from a QualNet simulation.

The simulated hardware parameters are based on the Senao IEEE $802.11 \mathrm{~b}$ device [20]. Data frames are transmitted at $11 \mathrm{Mb} / \mathrm{s}$ and contain a 1400 byte payload. ACK frames are returned at the same rate to illustrate the potential influence of ACK failures. The simulations use $10^{5}$ frame transmissions for each of $10^{3}$ initial random seeds. The transmission reliability of data and ACK frames are measured separately. Figure 7 shows decoding data frames at a given probability of success requires an RSS approximately $1 \mathrm{dBm}$ above that required to successfully decode an ACK frame in the QualNet simulation.

\section{B. Scenario Configuration}

The scenario contains between one and five relay candidates located as previously shown in Figure 1. This configuration is a pathological case for DAFMAC because relays 2, 3 and 5 have identical RSS values, which will deliberately induce retransmission collisions. This allows us to evaluate the validity of the proposed analytic retransmission model in terms of collision probability. The RSS and PDR values for all links are given in Table I.

\section{QualNet Simulation Comparison}

The QualNet simulation uses between one and five relays to illustrate the changes in protocol behaviour as more relays are added to the retransmission process. This increases the probability that a relay is able to retransmit, while also increasing the probability of collisions. Relays are added sequentially, so the set of three participating relays is $\mathcal{N}_{3}=\{1,2,3\}$. Each
TABLE I: Scenario link RSS and transmission PDR values

\begin{tabular}{ccccc}
\hline Node & $R S S_{s, i}(\mathrm{dBm})$ & $P_{D}(s, i)$ & $R S S_{i, d}(\mathrm{dBm})$ & $P_{D}(i, d)$ \\
\hline$s$ & 0 & 1.0 & -83 & 0.5 \\
1 & -72 & 1.0 & -82 & 0.79 \\
2 & -83 & 0.40 & -78 & 1.0 \\
3 & -83 & 0.40 & -78 & 1.0 \\
4 & -71 & 1.0 & -81 & 0.99 \\
5 & -73 & 1.0 & -78 & 1.0 \\
\hline
\end{tabular}

relay set configuration is simulated using $10^{3}$ random seeds to provide a statistically meaningful result.

The analytic retransmission model produces results nearly identical to the QualNet simulation, as shown in Figure 8. The median QualNet result is presented as a histogram. The confidence intervals represent the central $90 \%$ and are typically within of $1 \%$ of the median (barely visible in most cases). The diamond shaped point represents the corresponding analytically-generated result and is consistently inside the $90 \%$ confidence interval of the QualNet simulation.

It is worth pointing out that $\triangle$-MAC outperforms other models in a static environment where channel gain exhibits little temporal variation and one relay has a high probability of retransmission success, as shown in Fig. 8. This is due to the fact that $\triangle$-MAC is a centralised, globally coordinated, collision-free approach. It provides the performance benchmark to contention-based, decentralised, approaches, such as our proposed DAFMAC and preferred relay enabled DAFMAC protocols. However, $\triangle$-MAC requires extra signalling to coordinate the source, the relays and the destination. Moreover, practical transmission environments are usually not static due to mobility of the transmitters and receivers, as well as the surrounding radio reflectors. The DAFMAC protocols are shown to be of practical value in Section VI-C because they are more robust when there is a greater variability in the receiver decoding probability.

\section{Vi. AnAlytic Comparison of Representative COOPERATIVE RETRANSMISSION PROTOCOLS}

The proposed analytic retransmission model is independent from specific path loss or receiver decoding models. This section demonstrates the power of this feature by analytically comparing the ARQ, CMAC, DAFMAC, $\triangle$-MAC, and PRO retransmission schemes using two different receiver decoding models. Analysis is completed in the order of minutes rather than the days or weeks required for a full protocol simulation.

\section{A. Simulated Network Configuration}

Source and destination nodes are placed at fixed locations $130 \mathrm{~m}$ apart in the centre of a $250 \times 250 \mathrm{~m}$ area, as shown in Figure 9. Using the device parameters introduced in Section $\mathrm{V}$, the direct transmission success probability $P_{D}(s, d) \approx 0.5$. Path-loss is calculated using a log-normal model with a pathloss exponent of $n=2.6$, base distance $d_{0}=130 \mathrm{~m}$, and RSS values $R S S_{0}=-82.83 \mathrm{dBm}$ for the QualNet receiver. The base RSS value was normalised to $R S S_{0}=-85.3 \mathrm{dBm}$ for Judd and Steenkiste's empirical receiver to ensure an 


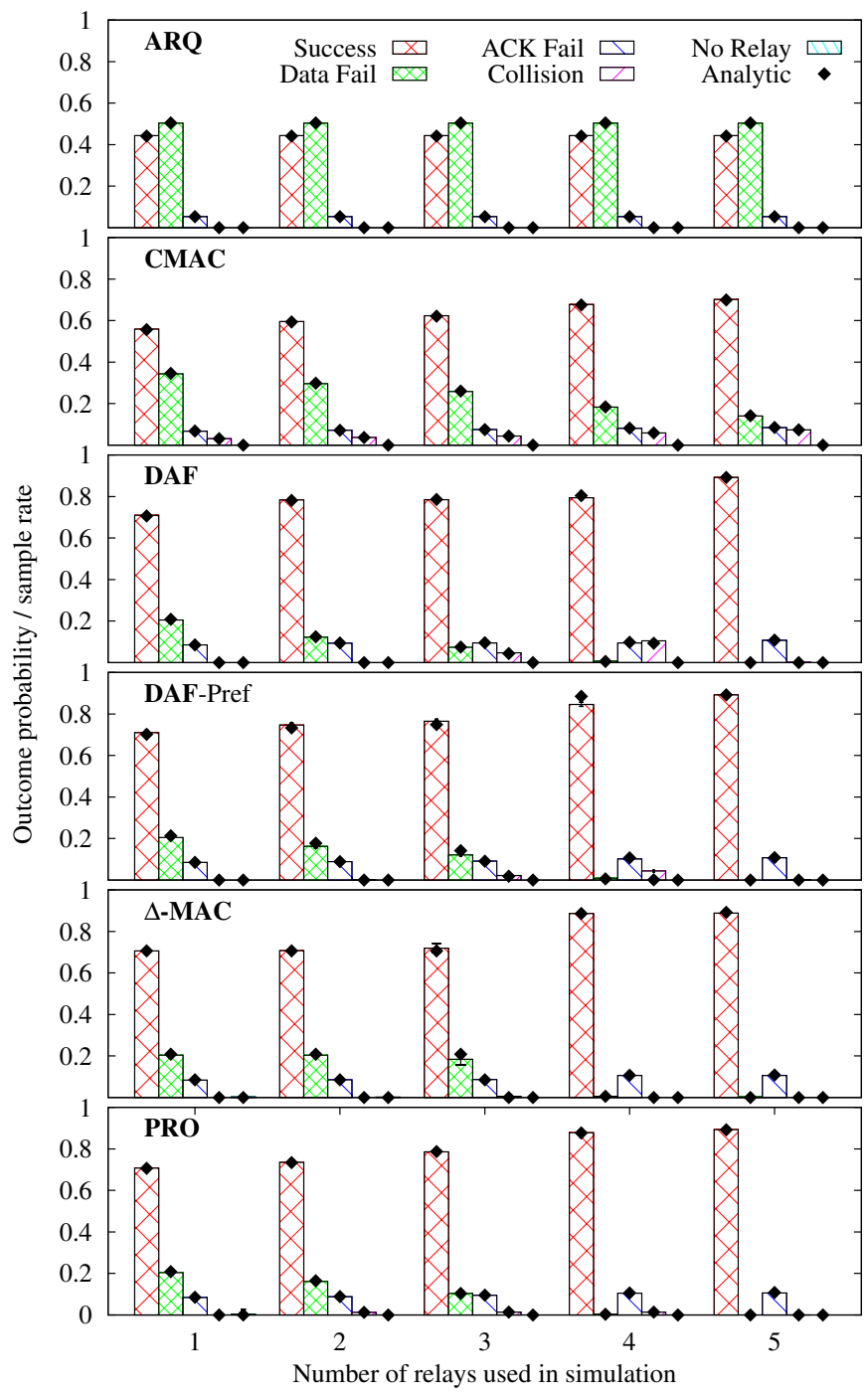

Fig. 8: The analytic model predicts nearly identical retransmission probabilities to those observed in an equivalent QualNet simulation using between one and five potential relays. $90 \%$ confidence intervals are barely visible due to the narrow spread of the results.

equivalent PDR at the nominated source to destination separation. Neighbour nodes are randomly distributed and the retransmission probability calculated for each algorithm for $10^{3}$ node sets for between one and five relays.

Each protocol uses a 32 slot contention window. DAFMAC uses $F_{\text {min }}=-85 \mathrm{dBm}$ for the QualNet model, $F_{\text {min }}=-88 \mathrm{dBm}$ for Judd and Steenkiste's receiver model, and $F_{\max }=F_{\min }+16 \mathrm{dBm}$. PRO uses a threshold of 0.95 .

\section{B. QualNet Receiver Model}

The mean retransmission outcome probability from the random placements is shown in Figure 10. For a fair comparison, we consider a contention-free environment in the case of the 802.11 ARQ protocol; in other words, no neighbouring relays contend with the source. This provides the best non-cooperative retransmission performance that the 802.11 ARQ protocol can achieve. Retransmission failures due to the

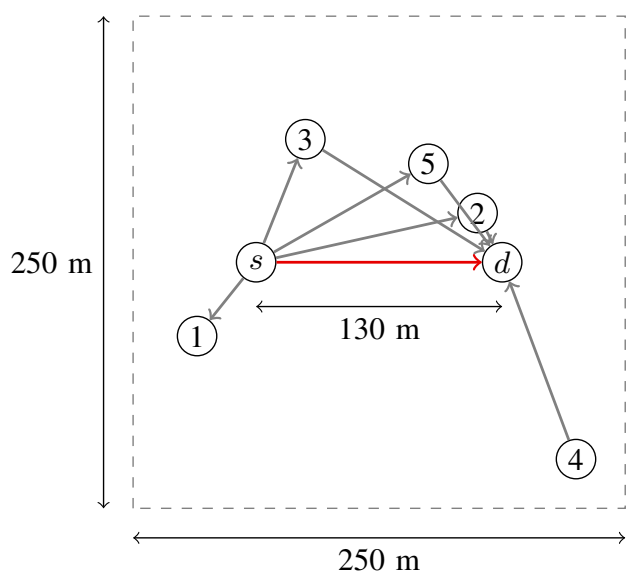

Fig. 9: Example scenario with fixed source and destination nodes with randomly placed neighbours

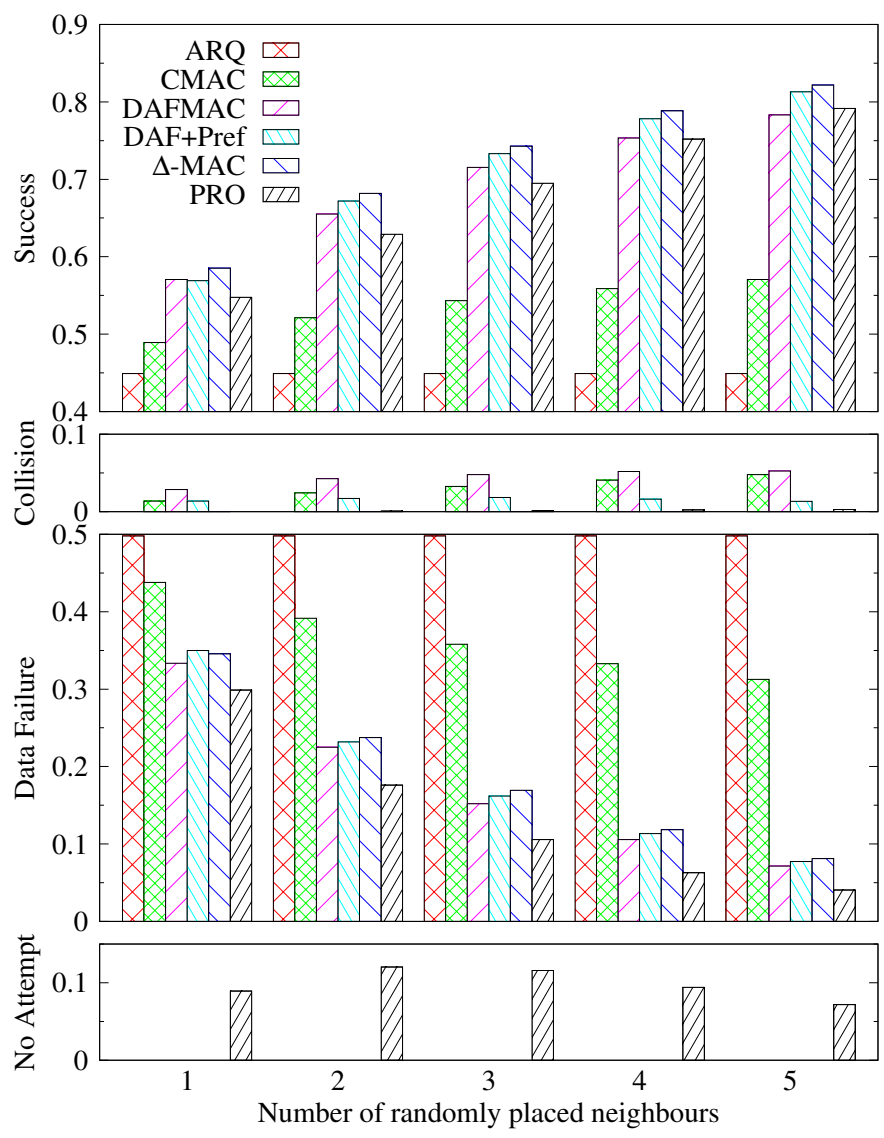

Fig. 10: The retransmission outcome probabilities using a random neighbour layout and the QualNet receiver probability model

source's failure to decode the ACK frame are independent of the cooperative protocol and are not shown.

As shown in the figure, the CMAC protocol performance is limited by the data failure rate because lower quality and higher quality relays are equally likely to win retransmission contention. DAFMAC with preferred relays consistently produces a lower collision rate and a higher retransmission probability than without preferred relays. $\triangle$-MAC has the 
highest retransmission success probability. This is because $\triangle$-MAC nominates the one relay with the best link quality. To achieve this, centralised coordination between the source, relays and the destination must be carried out with extra signalling requirement, as discussed in Section V. Finally, PRO performance is limited by the probability that no device meeting the performance criteria is able to enter retransmission contention.

\section{Judd and Steenkiste's Receiver Model}

An alternative receiver model can be constructed from empirical measurements of actual transceiver hardware performance under controlled conditions. Judd and Steenkiste used an FPGA-based hardware channel emulator to measure the rate that the receiver successfully decodes a frame for a given RSS value [21]. Their results indicate that real hardware often operates with a wider transition range (i.e. a slower transition from near-perfect performance to near-total failure) than the QualNet model (as shown in Figure 7), which will affect the behaviour and performance of the relay selection algorithm.

The empirical results reported by Judd and Steenkiste only include data frame decoding performance. ACK frame performance was not explicitly stated in [21], so an estimate based on a $3 \mathrm{dBm}$ offset from data frame transmission success probability was used (shown in Figure 7 as Estimated $(A)$ ). The $3 \mathrm{dBm}$ offset is based on the expected frame error rate for ACK frames calculated from the ACK frame size and the post-FEC bit error rate inferred from the data frame error rate. This gives results in a larger gap between data frame and ACK frame decoding success probability compared to the QualNet model (approximately $1 \mathrm{~dB}$ ), however in the absence of experimental data, it will suffice for this evaluation of relative protocol performance (as all protocols are evaluated with the same receiver model).

The mean retransmission outcome probability from the random placements is shown in Figure 11. Again, retransmission failures due to the source not decoding the ACK frame are independent of the cooperative protocol and are not shown.

The same general trends are also observed with the Judd and Steenkiste receiver model as with the QualNet radio. However, the DAFMAC retransmission success probability is improved and approximately equals that of $\triangle$-MAC, which is effectively the ideal result for a single relay system. This is because DAFMAC allows all relays to contend for retransmission and the greater spread of decoding success probability with respect to RSS in Judd and Steenkiste's receiver model improves the likelihood that the DAFMAC distributed relay selection algorithm will result in a single node winning access to the channel and relaying the frame. Conversely, $\triangle$-MAC and PRO use only a subset of relays for retransmission; reducing the size of the relay set decreases the probability one of these relays will successfully retransmit, although it also reduces the probability of a collision during retransmission attempts.

This illustrates a key feature of the analytic retransmission model - that retransmission performance of protocols depends on the receiver model used. Changing the receiver response in this analytic model is trivial, yet may not be possible in

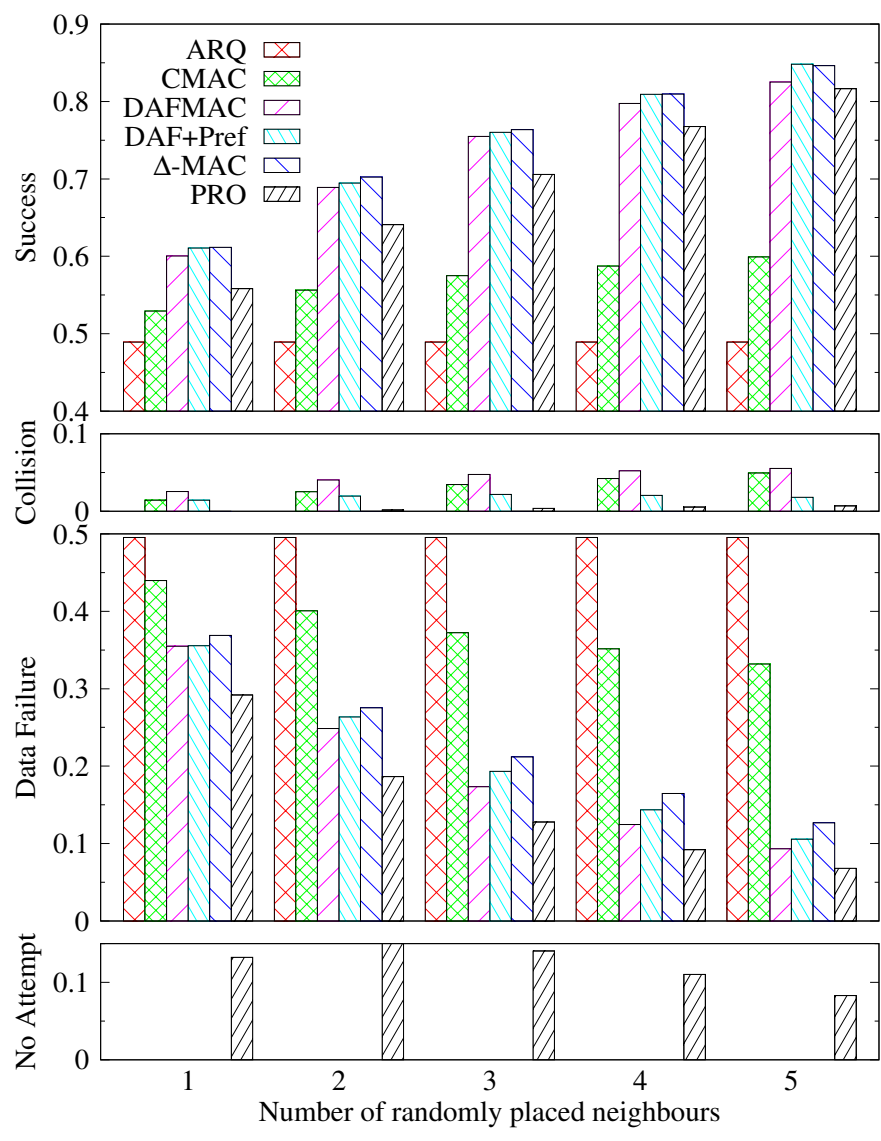

Fig. 11: The retransmission outcome probabilities using a random neighbour layout and Judd and Steenkiste's empirical receiver probability model [21]

simulation packages. This specific case highlights the robustness of DAFMACs distributed contention algorithm, which outperforms $\triangle$-MACs contention-fee algorithm, when using an alternate receiver model.

\section{APPLICATIONS AND DISCUSSIONS}

Our proposed analytic model can be used as a prediction tool, and operated in real-time to select adequate cooperative protocols adapting to changing network conditions. Specifically, the source or the destination can use the model to calculate the collision and success probabilities based on the instant relay ranking or scoring. It then picks up an adequate cooperative protocol, notifies the relays of the protocol, and therefore keeps the network operating in an efficient way.

Our model is also key to model the realistic network setting where every node can play the roles of source/relay/destination and switch between the roles at any time. Existing models developed so far are unable to characterise such a network setting, because the network is affected by a number of parameters, such as queue length, priority type, back-off window size, and network scale. Markov models have been developed to capture these parameters for each individual node [22][24]; while the model of the entire network needs to be established on the collision probability which connects the Markov processes of the individual nodes [22]. Modelling the 
cooperative retransmissions for a pair of source and destination (as described in Section III) is crucial to characterise the collisions that the cooperative retransmissions bring into the network. In this sense, our proposed model is key to model the more realistic network setting.

\section{CONCLUSION}

In this paper, we proposed a general model which can evaluate the distributed, contention-based, cooperative retransmissions of every data frame. We also proposed a Markov extension of our model to characterise the dependency between retransmissions, which enables us to analyse continuous retransmissions of successive frames. Our model is validated with a range of retransmission protocols, including $A R Q$, CMAC, DAFMAC, $\triangle$-MAC, and PRO. Confirmed by QualNet simulations, the proposed model can analytically predict the probabilities of cooperative retransmissions with an accuracy of $\pm 1 \%$. This enables direct comparisons between cooperative retransmission protocols, without the need to implement the full protocol in a state-based simulator.

\section{APPENDIX}

\section{Derivation of (13)}

A relay wins contention if it is the first (and only) node whose back-off timer reaches zero. Consider the contending set $\mathcal{N}_{c}$; node $i$ wins contention if all other timers expire after its timer, hence the probability that $i$ wins contention in time slot $t$ is:

$$
\begin{aligned}
\operatorname{Pr}\left\{i \text { wins, } t \mid i \in \mathcal{N}_{c}\right\} & =p_{i t}(t) \prod_{j \in \mathcal{N}_{c} \backslash\{i\}} p_{j a}(t) \\
& =W_{i}\left(\mathcal{N}_{c}, t\right)
\end{aligned}
$$

Extending (24), the probability that node $i$ wins contention in any time slot is:

$$
\operatorname{Pr}\left\{i \text { wins } \mid i \in \mathcal{N}_{c}\right\}=\sum_{t=0}^{T_{\max }-1} W_{i}\left(\mathcal{N}_{c}, t\right)
$$

The probability that relay $i$ wins contention, retransmits the data frame to $d$ and $s$ receives the ACK is therefore:

$\operatorname{Pr}\left\{i\right.$ succeeds $\left.\mid i \in \mathcal{N}_{c}\right\}=\sum_{t=0}^{T_{\max }-1} W_{i}\left(\mathcal{N}_{c}, t\right) P_{D}(i, d) P_{A}(d, s)$

The probability of any contending node (from the contending set $\mathcal{N}_{c}$ ) successfully forwarding the frame is:

$$
\operatorname{Pr}\left\{\text { success } \mid \mathcal{N}_{c}\right\}=\sum_{i \in \mathcal{N}_{c}} \sum_{t=0}^{T_{\max }-1} W_{i}\left(\mathcal{N}_{c}, t\right) P_{D}(i, d) P_{A}(d, s)
$$

Let $\mathcal{S}_{c}$ be the set of all possible combinations of contending relays and $\mathcal{N}_{c}$ be a specific contending set, such that $\mathcal{N}_{c} \subseteq \mathcal{S}_{c}$. The probability of a specific set $\mathcal{N}_{c}$ having received the source frame and contending is:

$$
p_{\mathcal{N}_{c}}=\prod_{i \in \mathcal{N}_{c}} P_{D}(s, i) \prod_{i \in \mathcal{N}_{p} \backslash \mathcal{N}_{c}}\left(1-P_{D}(s, i)\right)
$$

From (25) and (26), the probability that any node from any contending relay set successfully retransmits the frame to the destination and the ACK is successfully received is therefore as given by (13).

\section{REFERENCES}

[1] A. Bletsas, H. Shin, and M. Z. Win, "Cooperative communications with outage-optimal opportunistic relaying," IEEE Trans. Wireless Commun., vol. 6, no. 7, pp. 3450-3460, Sep. 2007.

[2] P. Liu, Z. Tao, and S. Panwar, "A cooperative MAC protocol for wireless local area networks," in Proc. IEEE ICC, Seoul, Korea, May 2005, pp. 2962-2968

[3] B. Sen, J. Guo, X. Zhao, and S. Jha, "ECTX: A high-throughput path metric for multi-hop wireless routing exploiting MAC-layer cooperative retransmission," in Proc. IEEE WoWMoM, San Francisco, USA, Jun. 2012, pp. 1-9.

[4] P. A. Anghel and M. Kaveh, "On the performance of selection cooperation ARQ," in Proc. IEEE ICC, Dresden, Germany, Jun. 2009, pp. $1-6$.

[5] B. Hagelstein, M. Abolhasan, D. Franklin, and F. Safaei, "An efficient opportunistic cooperative diversity protocol for 802.11 networks," in Proc. ACM IWCMC, Caen, France, Jul. 2010, pp. 417-421.

[6] M.-H. Lu, P. Steenkiste, and T. Chen, "Opportunistic retransmission in WLANs,” IEEE Trans. Mobile Comput., vol. 11, no. 12, pp. 1953-1969, Dec. 2012.

[7] Z. Wang, C. Li, and Y. Chen, "Local cooperative relay for opportunistic data forwarding in mobile ad-hoc networks," in Proc. IEEE ICC, Ottawa, Canada, Jun. 2012, pp. 1-6.

[8] L. Xiong, L. Libman, and G. Mao, "Optimal strategies for cooperative MAC-layer retransmission in wireless networks," in Proc. IEEE WCNC, Las Vegas, USA, Mar. 2008, pp. 1495-1500.

[9] N. S. Shankar, C.-T. Chou, and M. Ghosh, "Cooperative communication MAC (CMAC) - a new MAC protocol for next generation wireless LANs," in Proc. IEEE ICWNCMC, Maui, USA, Jun. 2005, pp. 1-6.

[10] N. Agarwal, D. ChanneGowda, L. N. Kannan, M. Tacca, and A. Fumagalli, "IEEE 802.11 b cooperative protocols: A performance study," in NETWORKING. Springer, 2007, pp. 415-426.

[11] C.-T. Chou, J. Yang, and D. Wang, "Cooperative MAC protocol with automatic relay selection in distributed wireless networks," in Proc. IEEE PerCom, White Plains, USA, Mar. 2007, pp. 526-531.

[12] J. Alonso-Zárate, E. Kartsakli, C. Verikoukis, and L. Alonso, "Throughput analysis of a medium access control protocol for a distributed cooperative arq scheme in wireless networks," in Proc. IEEE GLOBECOM, New Orleans, USA, Dec. 2008, pp. 1-5.

[13] M. Khalid, Y. Wang, I. ho Ra, and R. Sankar, "Two-relay-based cooperative MAC protocol for wireless ad hoc networks," in IEEE Trans. Veh. Technol., vol. 60, no. 7, Sep. 2011, pp. 3361-3373.

[14] M. Dianati, X. Ling, K. Naik, and X. Shen, "A node-cooperative ARQ scheme for wireless ad hoc networks," IEEE Trans. Veh. Technol., vol. 55, no. 3, pp. 1032-1044, May 2006.

[15] T. Issariyakul and E. Hossain, "Performance modeling and analysis of a class of ARQ protocols in multi-hop wireless networks," IEEE/ACM Trans. Netw., vol. 5, no. 12, pp. 3460-3468, Dec. 2006.

[16] IEEE Computer Society, IEEE Std. 802.11-2007 - Part 11: Wireless LAN Medium Access Control (MAC) and Physical Layer (PHY) Specifications. IEEE Standards Associations, Jun. 2007.

[17] B. Hagelstein, M. Abolhasan, D. Franklin, and F. Safaei, "Improving fairness in IEEE 802.11 networks using MAC layer opportunistic retransmission," Elsevier Comput. Netw., no. 17, pp. 3410-3427, Dec. 2013.

[18] W. J. Stewart, Introduction to the Numerical Solution of Markov Chains. Princeton University Press, 1994.

[19] Scalable Network Technologies, Inc., QualNet 4.5 Users Guide, 2008.

[20] Senao Networks, Inc, "Senao 2511CD Wireless LAN Card User Guide," Available online: http://www.senao.com/images/www.senao. com_English/2511CD-EXT2_UserManual_20020918.pdf, 2002.

[21] G. Judd and P. Steenkiste, "Characterizing 802.11 wireless link behavior," Wireless Networks, vol. 16, no. 1, pp. 167-182, Jan. 2010.

[22] G. Bianchi, "Performance analysis of the IEEE 802.11 distributed coordination function," IEEE J. Sel. Areas Commun., vol. 18, no. 3, pp. 535-547, Mar. 2000.

[23] K. Ghaboosi, B. H. Khalaj, Y. Xiao, and M. Latva-aho, "Modeling IEEE 802.11 DCF using parallel space-time Markov chain,” IEEE Trans. Veh. Technol., vol. 57, no. 4, pp. 2404-2413, Jul. 2008. 
[24] R. P. Liu, G. J. Sutton, and I. B. Collings, "A new queueing model for QoS analysis of IEEE 802.11 DCF with finite buffer and load," IEEE Trans. Wireless Commun., vol. 9, no. 8, pp. 2664-2675, Aug. 2010.

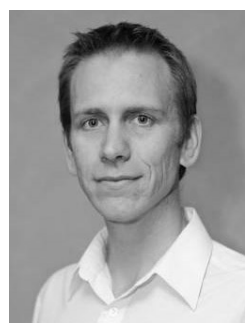

Brett Hagelstein received the B.E. degree from the University of Wollongong in 2007 and the Ph.D. degree from the University of Technology, Sydney in 2013. He is currently a Research Associate at the University of Technology Sydney. His research interests include cooperative algorithms in wireless networks, routing protocols and embedded systems.

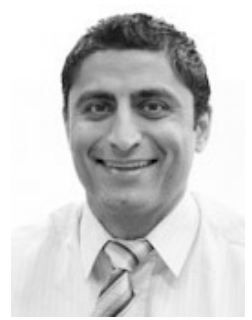

Mehran Abolhasan completed his B.E in Computer Engineering and $\mathrm{PhD}$ in Telecommunications on 1999 and 2003 respectively at the University of Wollongong. He has led several major R\&D projects in various areas of wireless communications since 2003 and has authored over 60 international publications and has won over one million dollars in research funding. His current research interests are in wireless mesh, 4th generation cooperative networks and body-area/sensor networks. He is currently an Associate Professor at the University of Technology Sydney and a Senior Member of IEEE.

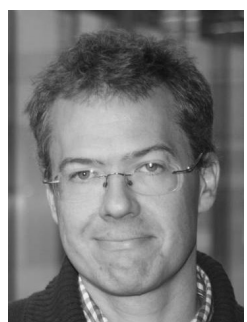

Daniel Franklin completed his $\mathrm{PhD}$ in Electrical Engineering - "Enhancements to Channel Models, DMT Modulation and Coding for Channels Subject to Impulsive Noise", at the University of Wollongong in 2007. He also holds a Bachelor of Engineering (electrical) - Honours I, University of Wollongong, 1995-1998 and has been a member of the Institution of Electrical and Electronics Engineers since 1998 . He is currently a Senior Lecturer in the Faculty of Engineering and Information Technology at the University of Technology, Sydney. His research and commercial interests include wireless networks (routing protocols, mesh networking, modulation and coding), medical radiation physics (solidstate detectors for PET, gamma-ray dosimetry), embedded and real-time systems, and analogue and digital electronics.

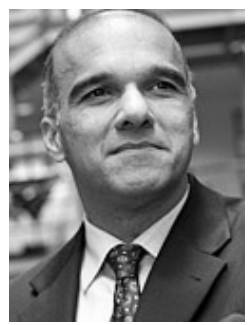

Farzad Safaei graduated from the University of Western Australia with the degree of Bachelor of Engineering (Electronics) and obtained his $\mathrm{PhD}$ in Telecommunications Engineering from Monash University, Australia. Currently, he is the Professor of Telecommunications Engineering and Managing Director of ICT Research Institute at the University of Wollongong. Before joining the University of Wollongong, he was the Manager of Internetworking Architecture and Services Section in Telstra Research Laboratories.

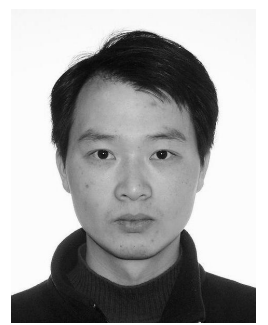

Wei Ni (M'09) received the B.E. and Ph.D. degrees in Electronic Engineering from Fudan University, Shanghai, China, in 2000 and 2005, respectively. Currently he is a Senior Research Scientist in the Digital Productivity and Service (DP\&S) Flagship, CSIRO, Australia. Prior to this he was a Research Scientist and Deputy Project Leader at the Bell Labs R\&I Center, Alcatel-Lucent (2005-2008), and a Senior Researcher at Devices R\&D, Nokia (20082009). His research interests include MIMO, Relay Mesh, RRM, and Scheduling. He serves as an Editorial Board Member for Hindawi Journal of Engineering since 2012. 